\title{
FREQUENCY-BASED FEATURES FOR BEARINGS CONDITION MONITORING EXTRACTED WITH GOERTZEL ALGORITHM
}

\author{
Daniel Cordoneanu ${ }^{1}$, Constantin Nitu ${ }^{1}$ \\ ${ }^{1}$ Department of Mechatronics and Precision Mechanics, University "Politehnica" of Bucharest \\ Splaiul Independentei 313, Bucharest 060042 \\ daniel.cordoneanu@stud.mec.upb.ro
}

\begin{abstract}
Condition monitoring has been a field of interest in the latest period, especially predictive maintenance, given the advances in artificial intelligence algorithms available in a great number of libraries. In the industrial sector, condition monitoring and fault diagnosis play a very important role in order to avoid as much as possible downtime. Usually, rotating motors are involved in the actuation of the machines used in industry; therefore bearings are an important part of the kinematic chain. Given that faults in bearings can be detected in the frequency spectrum at frequencies that can be mathematically computed based on the mechanical geometry, this paper proposes an approach to extract features for machine learning algorithms based on these computed frequencies and their harmonics. Since only a few frequencies are needed, the Goertzel algorithm can be used instead of the discrete Fourier transform to give a computational boost and have the feature extraction algorithm available on embedded systems.
\end{abstract}

Keywords: Goertzel, Bearings, Condition Monitoring, Frequency Features, FFT.

\section{Introduction}

Condition monitoring gets more and more attention nowadays as the hardware and software advancements provide more power and more efficient ways to assess whether a machine has a fault or not. Of course, condition monitoring and fault diagnosis mean, besides fault detection, an identification of the fault and the part of the machine that is broken. The importance of detecting an early fault can save a lot of money, as maintenance can be properly scheduled and the downtime of a machine can be decreased.

In [1], Natu describes the number of faults that can appear in a rotating machine per part: $40 \%$ bearing faults, $38 \%$ stator faults, $10 \%$ rotor faults, others $12 \%$. Since the majority of the faults occur in bearings, it is of high importance to have these parts monitored, as an issue in these rolling elements is usually provoked by another component or suggests improper lubrication or wearing-off, in which case the bearing has to be repaired or replaced so that other components are not affected in time. In literature there are many algorithms described which usually use the spectrum analysis on which different filter banks are applied, as in [2], where mel filter banks are used to extract features as mel cepstral coefficients that are later used in an algorithm that uses gaussian mixture models and kurtosis. Kurtosis is successfully used as well in [3] alongside envelope spectrum analysis.
In [4], Nabhan et al. summarize fault detection techniques for ball bearings and shows that vibration measurements and spectrum analysis techniques are the most useful tools for fault diagnosis in rolling bearings. Since most of these techniques rely on the Fast Fourier Transform (FFT), which in acquisition and signal processing devices is done as the Discrete Fourier Transform (DFT), a more efficient algorithm can be used to extract specific frequencies information that doesn't have to deal with spectral leakage and is not dependent of the number of points of the DFT.

\section{Bearing Fault Condition}

\subsection{Rolling bearing description}

A rolling bearing is a rotating machine element that enables the movement by reducing friction and handles the stress coming from the linked components. In [5], bearing fault spectral analysis as well as other methods are described. In section 4.3 , it is explained that a fault can be indicated if there is a peak at the fundamental fault frequency and the $2^{\text {nd }}$ harmonic of this frequency; also, if there is no peak at the fundamental fault frequency, but there are peaks in the next harmonics, then the fault is real. In figure 1 and figure 2, the geometric variables that help finding the fundamental fault frequencies are shown: 


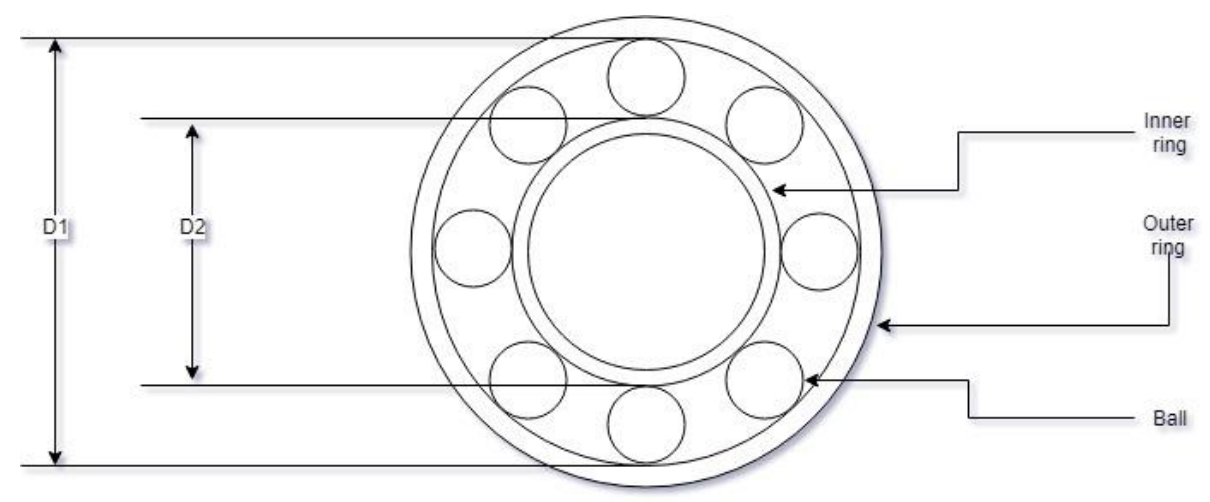

Figure 1: Bearing elements

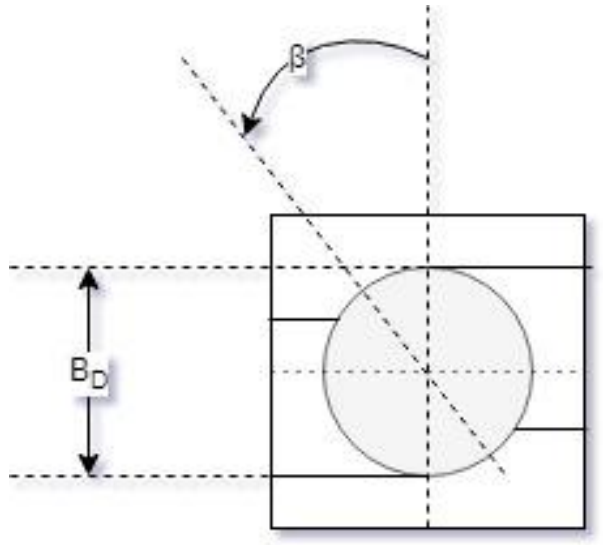

Figure 2: Bearing ball

\subsection{Types of faults in bearings}

Given the geometries in the above figures, in [5] the causes of the bearing failures are described: bad lubrication, heavier load than expected, wear off because of time, misplacement of shaft, etc. Initially, bearing fatigue results in shear stresses below the load-carrying surface. In time, these shears turn to cracks in the exterior surface and as the load goes over them, fragments are ripped apart from the bearing. This type of fault is usually assessed through vibrations since mechanical waves that are produced by the cracks get higher amplitude as time goes. Surface distress is another type of fault that can produce cracks and it's usually provoked by improper lubrication or heavier load.

To diagnose properly a bearing fault, the following bearing frequencies can be calculated:

- $\mathrm{F}_{\text {ord }}=$ Frequency Outer Race Defect

- Fird = Frequency Inner Race Defect

- $\mathrm{Fbd}_{\mathrm{b}}=$ Frequency Ball Defect

- $\mathrm{F}_{\mathrm{c}}=$ Frequency Cage

Based on figure 1, we can determine the following variable as the pitch diameter:

$$
P_{d}=\frac{D_{1}+D_{2}}{2}
$$

The above described frequencies can be computed as follows:

$$
\begin{aligned}
& F_{\text {ord }}=\frac{n f_{\text {rotation }}}{120}\left(1-\frac{B_{d} \cos (\beta)}{P_{d}}\right) \\
& F_{\text {ird }}=\frac{n f_{\text {rotation }}}{120}\left(1+\frac{B_{d} \cos (\beta)}{P_{d}}\right) \\
& F_{\text {bd }}=\frac{f_{\text {rotation }}}{60} * \frac{P_{d}}{B_{d}}\left[1-\left(\frac{B_{d} \cos (\beta)}{P_{d}}\right)^{2}\right] \\
& F_{c}=\frac{f_{\text {rotation }}}{120}\left(1-\frac{B_{d} \cos (\beta)}{P_{d}}\right)
\end{aligned}
$$

Where:

- $\mathrm{n}$ is the number of balls

- $\mathrm{B}_{\mathrm{d}}$ is the ball diameter

- $\beta$ is the contact angle

- $\mathrm{f}_{\text {rotation }}$ is the rotation frequency of the shaft in RPM

Further, these base frequencies and their multiples will be of interest when computing the DFT magnitude using the Goertzel algorithm.

\section{Goertzel Algorithm}

\subsection{Standard Goertzel algorithm}

As shown in [6] by Sysel \& Rajmic, the original algorithm described by Goertzel in [7] that computes the DFT term of a signal $x[n]$ with length $N$ can be treated as a discrete linear convolution between the signal $x[n]$ and $h_{k}[n]$ so that if the result of the convolution is $\mathrm{y}_{\mathrm{k}}[\mathrm{n}]$, then:

$$
y_{k}[m]=\sum_{n=0}^{\mathbb{N}-1} x[n] e^{j 2 \pi k \frac{m-n}{N}} u[m-n]
$$

Equation (6) is derived from the fact that $\mathrm{h}_{\mathrm{k}}$ can be expressed as:

$$
h_{k}[l]=e^{j 2 \pi k \frac{\mathbb{N}}{N}} u[l]
$$

As Sysel and Rajmic further show in their paper, equation (6) can be treated as an IIR linear system with the impulse response $h_{k}[n]$; the output sample $\mathrm{N}$ of this filter is the desired DFT component. Described as a second order IIR filter using 
differences, the equation of the filter can be written using state variables:

$$
s[n]=x[n]+2 \cos \left(\frac{2 \pi k}{N}\right) s[n-1]-s[n-2]
$$

With the final output being:

$$
y_{k}[n]=s[n]-e^{-j \frac{2 \pi k}{N}} s[n-1]
$$

As the author himself mentioned, only $\mathrm{N}$ multiplications and $2 \mathrm{~N}$ additions are needed.

\subsection{Goertzel algorithm compared to the DFT}

There are multiple advantages of the Goertzel algorithm over the DFT under certain conditions. If $\mathrm{K}$ $<4 / 7 \mathrm{~N}$ [6], where $\mathrm{K}$ is the number of frequencies in which we're interested in, then the Goertzel algorithm is superior in computation speed to the DFT.

Another advantage of the Goertzel algorithm is that one can inspect a signal of length $\mathrm{N}$ without bothering that $\mathrm{N}$ is a power of 2 (case in which DFT is computationally fast).

Also, while inspecting the spectrum, based on the sampling frequency and the number of samples we don't always have the correct magnitude of a certain frequency since it can't be represented by the DFT due to spectral leakage (e.g. if we have a sampling frequency of $12 \mathrm{kHz}$ with 1024 number of points, the frequency per bin of the DFT would be $12 \mathrm{kHz} / 1024$ $=11.71 \mathrm{~Hz}$ per bin so that if we want to know the magnitude of the sinusoid with a period of $115 \mathrm{~Hz}$, this would be leaked to the neighboring frequency bins of $117.1 \mathrm{~Hz}$ and $105.39 \mathrm{~Hz}$ ).

\subsection{Generalized Goertzel algorithm}

With a small computation expense, in [6] a generalized Goertzel algorithm is proposed that can use $\mathrm{K}$ as a real number, not only as an integer. This is helpful in bearing fault diagnosis case because the frequencies computed will most probably be real numbers since the cosine function is involved and also division to different numbers of different quantities.

\section{Feature Extraction and Results}

\subsection{Proposed algorithm}

The proposed algorithm in this paper is a very simple one, yet it's one that can be computationally efficient and can be used to extract features for a machine learning algorithm, be it a multi-class identifier like a neural network or just an anomaly detection algorithm using gaussian distribution to assess whether the values recorded in the harmonics of the fault frequencies are part of the side probabilities of the normal distribution. The algorithm can be described in the following steps:

- Find the rotation frequency of the system that rotates the bearing

- Find the specifications of the bearing to be monitored

- Compute the frequencies described in (2), (3), (4), (5)

- Compute the harmonics of the frequencies up to 10 harmonics (as described [5], the magnitudes should rise in up to the $4^{\text {th }}$ harmonic, but it's echoes should be visible in the higher spectrum as well)

- Apply the Goertzel algorithm on the computed frequencies to extract the DFT components and computer the squared length of the vector described by the components

- Split the computed harmonics per fault or use them all as features, depending on the chosen machine learning algorithm

In figure 3 we can see the flowchart for the algorithm.

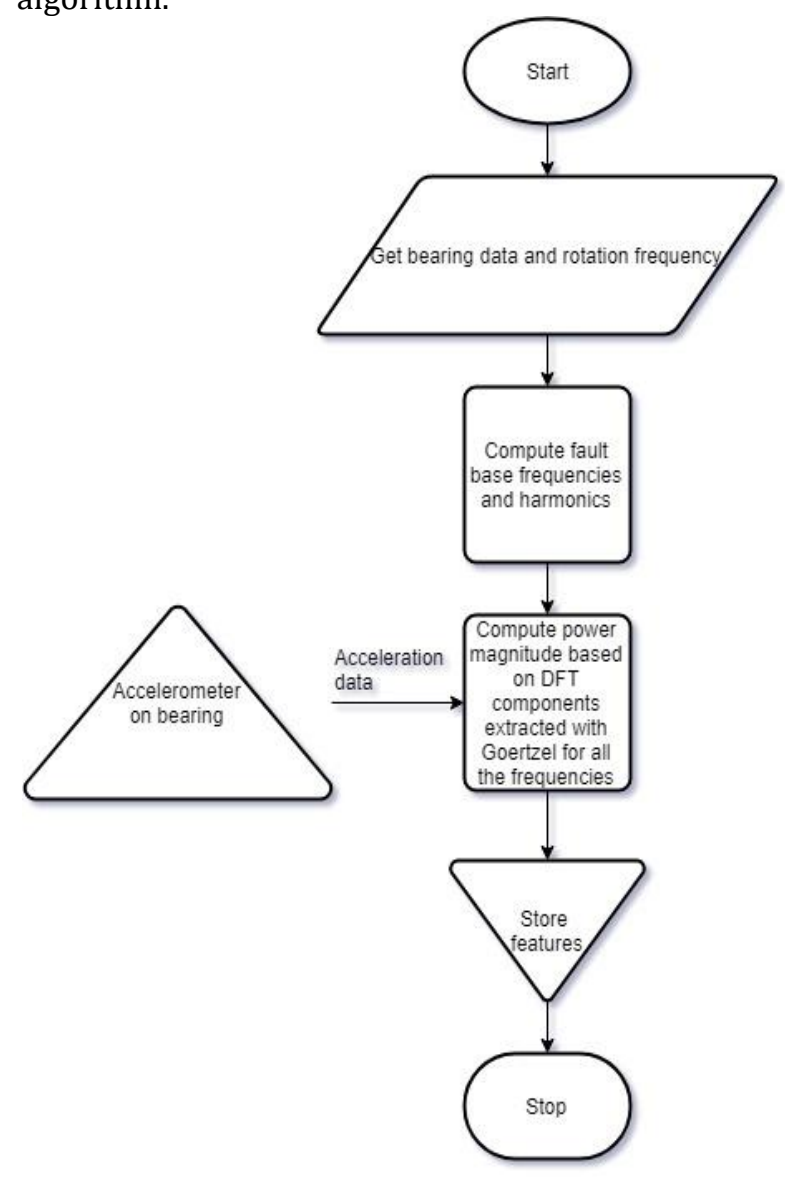

Fig. 3. Algorithm flowchart

\subsection{Experimentation}

To check the algorithm's results, the data obtained in the experimentation done by Lou and Loparo in [8] was used. The data comes from Rockwell Science Center and it's available on [9]. For the experiments, a $2 \mathrm{hp}$ Reliance Electric motor was 
used and acceleration data was fetched from the motor bearings.

Faults were made to the motor bearings using electro-discharge machining (EDM). Faults diameters ranged from 0.007 to 0.040 inches and data acquisition was made at $12 \mathrm{kHz}$ for bearings in normal conditions and faulty conditions at different speeds of the motor. Also, tables with fault frequencies are available directly on this resource so that the computations were easily made. Two bearings were used on the drive end and fan end.

The proposed algorithm will be applied on the drive end bearing (6205-2RS JEM SKF, deep groove ball bearing), for a motor speed of $1797 \mathrm{rpm}$.

In table 1, the bearing's data is presented and in table 2 the fault frequencies for $1797 \mathrm{rpm}$ :

Table 1. Bearing data in $\mathrm{mm}$

\begin{tabular}{ccclc}
\hline $\begin{array}{l}\text { Inside } \\
\text { diameter }\end{array}$ & $\begin{array}{c}\text { Outside } \\
\text { diameter }\end{array}$ & Thickness & $\begin{array}{l}\text { Ball } \\
\text { diameter }\end{array}$ & $\begin{array}{l}\text { Pitch } \\
\text { diameter }\end{array}$ \\
\hline 25 & 52 & 15 & 7.94 & 39.03 \\
\hline
\end{tabular}

Table 2. Bearing fault frequencies $(\mathrm{Hz})$ for $1797 \mathrm{rpm}$

\begin{tabular}{cccc}
\hline$F_{\text {ord }}$ & F $_{\text {ird }}$ & $F_{b d}$ & $F_{\mathrm{c}}$ \\
\hline 107.36 & 162.18 & 141.16 & 11.92 \\
\hline
\end{tabular}

To be noted that in the data analysis, since there were no cage faults, the $F_{c}$ was ignored.

\subsection{Results}

The goal in fault diagnosis is to detect the fault as early as possible, so the important data is the one for the faults with the diameter of 0.007 inches. However, also the 0.014 faults were added for comparison.

In the following figures, ball fault, inner race fault and outer race fault data is shown in comparison with the normal data. The original data was split into batches of 5000 points each which is the equivalent of $0.41 \mathrm{~ms}$. Here we can see that the Goertzel algorithm has no restriction regarding the number of points, nor the computed frequencies.

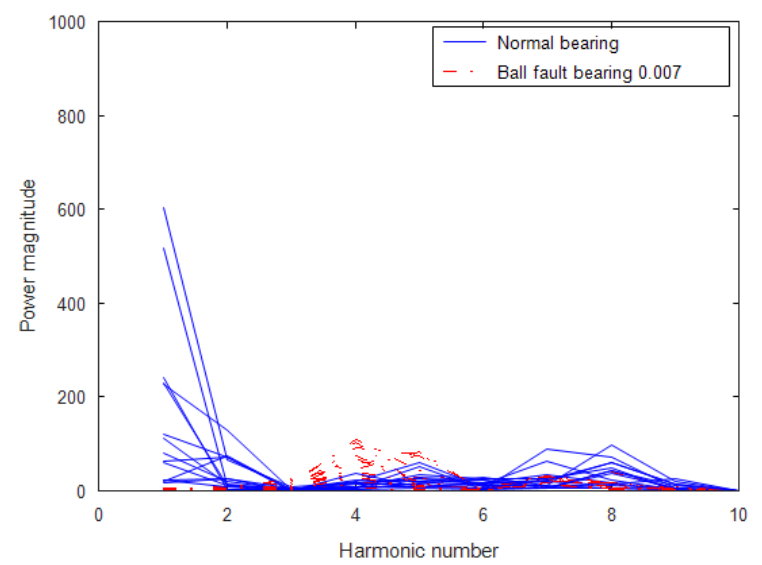

Fig. 4. Ball fault and normal data in 0.007 inches fault diameter

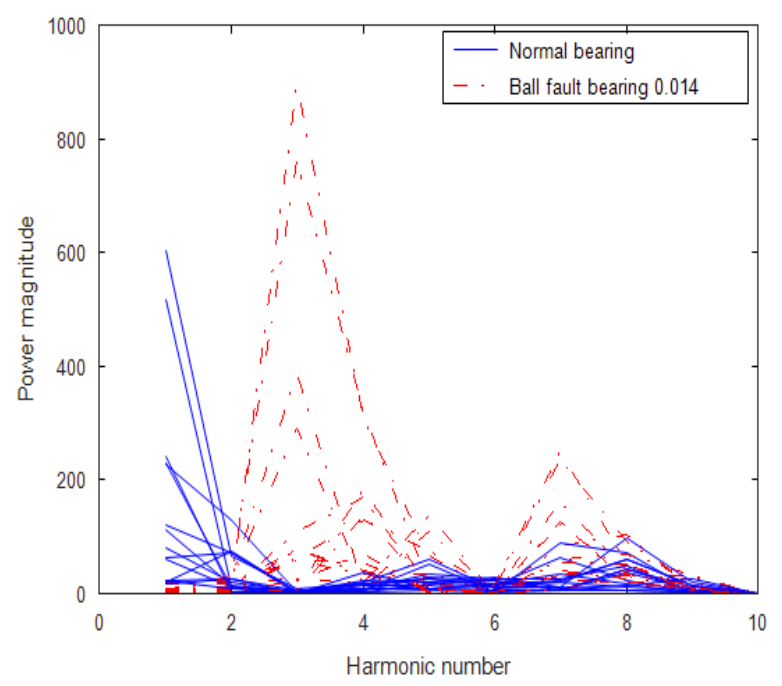

Fig. 5. Ball fault and normal data in 0.014 inches fault diameter

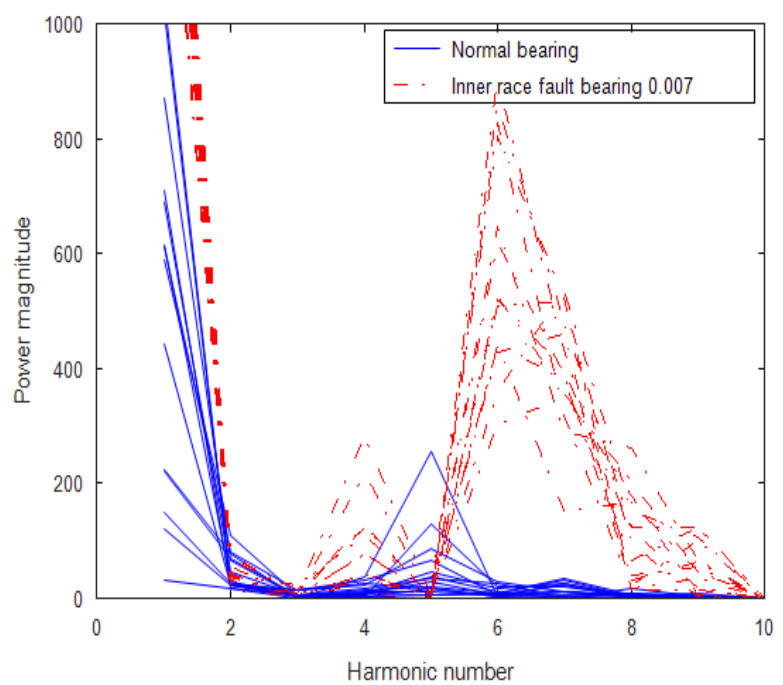

Fig. 6. Inner race fault and normal data in 0.007 inches fault diameter

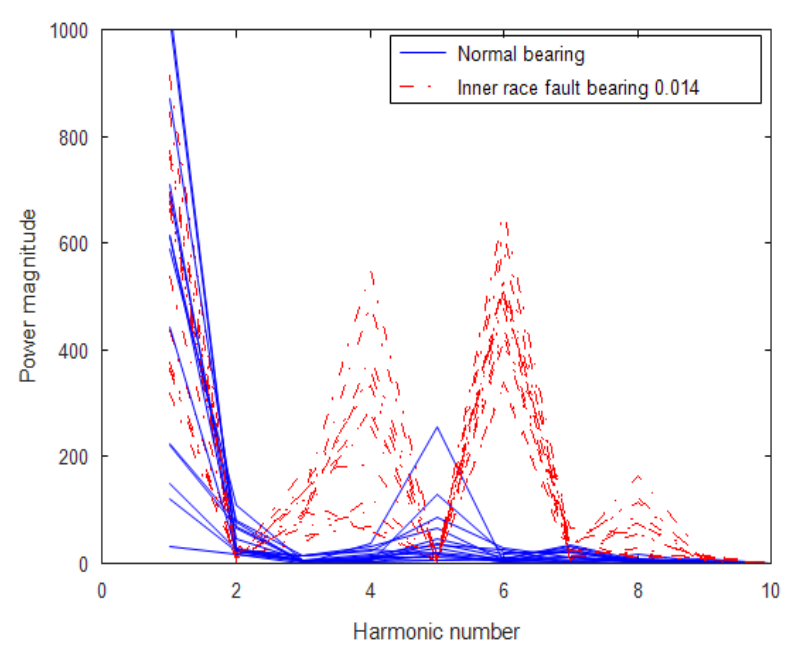

Fig. 7. Inner race fault and normal data in 0.014 inches fault diameter 


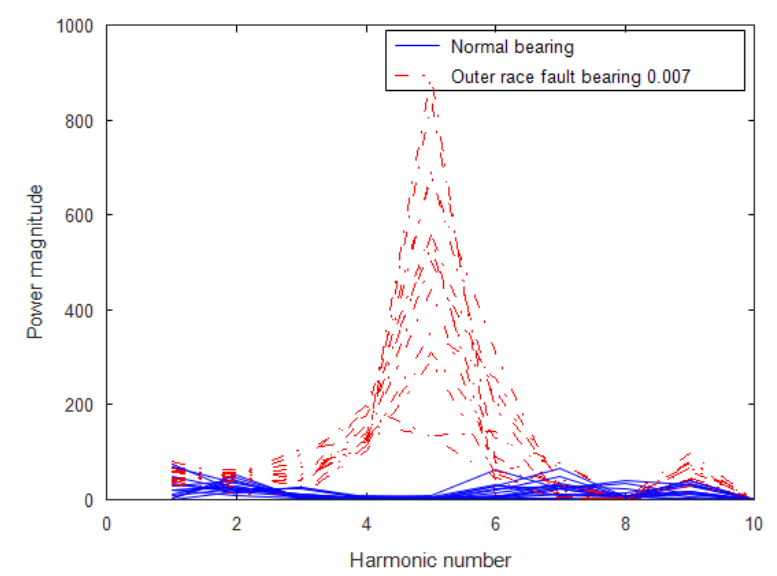

Fig. 8. Outer race fault and normal data in 0.007 inches fault diameter

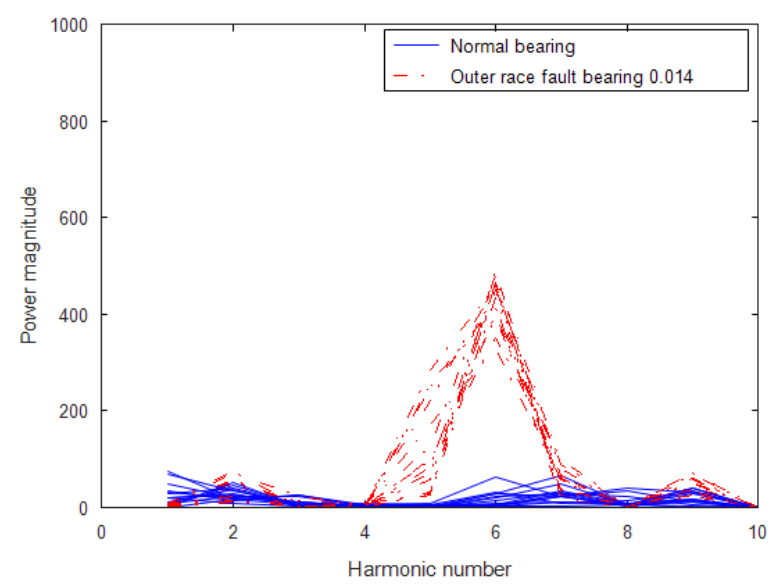

Fig. 9. Outer race fault and normal data in 0.014 inches fault diameter

\subsection{Evaluating the features with a simple probabilistic model}

For evaluating the features, an anomaly detection algorithm is proposed to check if these features have a Gaussian distribution.
By computing the mean vector and the covariance matrix, the probability density function of the multivariate Gaussian distribution can be computed.

Using only the normal data to train the model, the probability histogram would look like in figure 10:

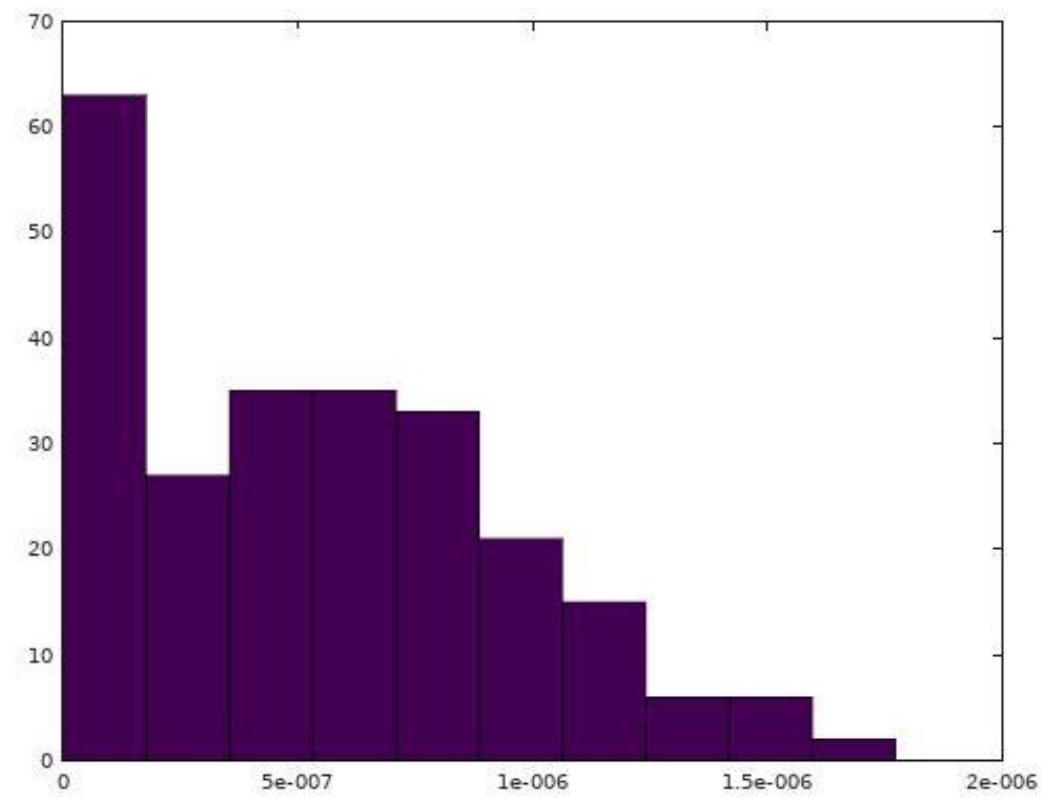

Fig. 10. Anomaly detection model 
As it can be seen, the features do not meet the requirements for a Gaussian distribution, so therefore just this simple algorithm would not do to use these features out of the box.

\section{Conclusions and Future Work}

As it can be seen in figures 4 and 5 faults can be separated from the normal behavior in multiple training examples with the mention that for this type of fault (ball fault), as the fault is small in diameter, the magnitude is very small compared to the 0.014 inches fault but it can be separated from the normal data magnitudes. For the other type of faults (visible in figures 6 to 9), the faulty data can be easily separable from the normal data.

Therefore we can conclude that the proposed algorithm can be used to extract features for fault diagnosis in bearings without using the DFT, but a more computational efficient algorithm. The same approach can be used for other mechanical parts where fault frequencies can be computed.

As it was shown in 4.4, using simple algorithms sometimes is not enough to model extract useful information from an already computational-efficient algorithm.

In the future, the features will be tested with different artificial algorithms in order to assess its accuracy based on the way the features are separated and classify what algorithms are worth using with this type of features.

\section{References}

[1] M. Natu, "Bearing Fault Analysis using Frequency and Wavelet Techniques," Int. J. Innov. Manag. Technol., 2012.

[2] F. V. Nelwamondo and T. Marwala, "Faults detection using Gaussian mixture models, melfrequency cepstral coefficients and kurtosis," in Conference Proceedings - IEEE International Conference on Systems, Man and Cybernetics, 2007.

[3] S. Wan, X. Zhang, and L. Dou, "Compound fault diagnosis of bearings using improved fast spectral kurtosis with VMD," J. Mech. Sci. Technol., 2018.

[4] A. Nabhan, N. M. Ghazaly, A. Samy, and M. M.O, "Bearing fault detection techniques - a review," Turkish J. Eng. Sci. Technol., 2015.

[5] SKF, "Bearings Spectrum Analysis," 2012.

[6] P. Sysel and P. Rajmic, "Goertzel algorithm generalized to non-integer multiples of fundamental frequency," EURASIP J. Adv. Signal Process., vol. 2012, no. 1, p. 56, Mar. 2012.

[7] G. Goertzel, "An Algorithm for the Evaluation of Finite Trigonometric Series," Am. Math. Mon., 2006.

[8] X. Lou and K. A. Loparo, "Bearing fault diagnosis based on wavelet transform and fuzzy inference," Mech. Syst. Signal Process., 2004.

[9] "Bearing Data Center." [Online]. Available: http://csegroups.case.edu/bearingdatacenter/ home. [Accessed: 02-Jun-2019]. 